\title{
Inmunopatogenia del psoriasis, evolución de la enfermedad, presentación clínica y tratamiento.
}

\section{Immunopathogenesis of psoriasis, evolution of the disease, clinical presentation and treatment}

Jorge Cañarte Alcivar. ${ }^{1}$, Vielka Jiménez García. ${ }^{2}$, Johan Muñoz Puertas. ${ }^{3}$, Ambar Luzardo Robles. ${ }^{4} \&$ Josue Delgado Villafuerte. ${ }^{5}$

\begin{abstract}
. https://doi.org/10.32/cienciadigital.v3i1.947

Psoriasis is a $\mathrm{T}$ cell-mediated chronic inflammatory disease of the skin. It affects genetically predisposed individuals and presents several subtypes. It is characterized by the presence of well-defined erythematous, scaly, irregular border plaque or lesions, affecting mainly the elbows, knees, scalp, and trunk. The HLACw6 allele of major histocompatibility system is related to the presence and severity of this disease. From the physiopathogenic viewpoint, psoriasis is a Th1- type immune disease in which the axle IL-23/Th17 is fundamental. Th17 cells produce proinflammatory cytokines (IL-17A, IL-17F, IL-22 and IL-26) which activate keratinocytes and cause hyperproliferation and increased production of proinflammatory cytokines and antimicrobial peptides
\end{abstract}

Keywords: psoriasis, immunopathogenesis, T cells, IFNa, plasmacytoid dendritic cells, Th17 cells.

\footnotetext{
${ }^{1}$ Universidad Técnica de Manabí, Facultad de Ciencias de la Salud, Ecuador, j.canarte.a@ gmail.com

${ }^{2}$ Universidad Técnica de Manabí, Facultad de Ciencias de la Salud, Ecuador, vjimenez6631@utm.edu.ec

${ }^{3}$ Universidad Técnica de Manabí, Facultad de Ciencias de la Salud, Ecuador, jmuñoz3898@utm.edu.ec

${ }^{4}$ Universidad Técnica de Manabí, Facultad de Ciencias de la Salud, Ecuador, aluzardo7848@utm.edu.ec

${ }^{5}$ Universidad Técnica de Manabí, Facultad de Ciencias de la Salud, Ecuador, jdelgado0961@utm.edu.ec
} 


\section{Resumen.}

La psoriasis es una enfermedad inflamatoria crónica de la piel mediada por células T que afecta a individuos con predisposición genética y presenta varios subtipos clínicos. Se caracteriza por la presencia de placas eritematosas bien definidas, escamosas y de bordes irregulares, que afectan fundamentalmente las regiones de los codos, las rodillas, el cuero cabelludo y el tronco. Desde el punto de vista inmunopatogenico, la psoriasis es una enfermedad inmune de tipo Th1, en la que es fundamental el eje IL-23/Th17. Las células Th17 producen las citosinas proinflamatorias (IL-17A, IL-17F, IL-22 e IL-26) que activan los queratinocitos y causan hiperproliferación y mayor producción de citosinas proinflamatorias y péptidos antimicrobianos, los que a su vez reclutan y activan otras células inmunes de la piel inflamada.

Palabras claves: psoriasis, inmunopatogenia, células T, IFNa, células dendríticas plasmacitoides, células Th17.

\section{Introducción.}

"La psoriasis se conoce como una enfermedad autoinmune en la cual factores tanto ambientales como genéticos tienen un rol importante" (Asadullah K, 1999).

En pocas palabras no se puede afirmar con certeza el carácter auto inmunitario de la enfermedad por la ausencia de auto antígeno y de auto anticuerpos específicos.

El paciente con psoriasis es vulnerable genéticamente a padecerla, a este evento se une un defecto inmunológico y biomolecular subyacente, que aún no está precisado plenamente.

La etiopatogenia es compleja y hoy todavía no del todo conocida. La psoriasis es una enfermedad hereditaria, aunque no se conoce el modo de herencia. Esta patología va a afectar a individuos con cierta predisposición genética y va a estar mediada por células T. En su cuadro inflamatorio del psoriasis interceden, tanto elementos de la respuesta inmune innata, como de elementos de la respuesta inmune adaptativa. Entre los primeros se encuentran las células dendríticas, presentadoras de antígenos, los neutrófilos, los queratinocitos, macrófagos y las células Natural Killer (NK); entre los segundos, se hallarán los linfocitos T CD4+ y los linfocitos T CD8+. (Baker BS, et al,2006)

Se va a encontrar en varias presentaciones, pero la más común es la Psoriasis en placas, que está caracterizada con la aparición de lesiones cutáneas de tamaño variables, enrojecidas y recubiertas de escamas de grosor variable. Estas lesiones pueden aparecer en cualquier lugar del cuerpo, pero tiende a afectar más comúnmente los codos, el cuero cabelludo, rodillas y espalda. (Dermatology, 2016).

"La prevalencia es de 1-6\% en la población mundial, afecta a hombres y mujeres por igual, se presenta en cualquier edad, pero su aparición predomina entre los 18 y 50 años, generalmente es 
más habitual en personas de raza blanca, que pertenecen a piel tipo I y II según la clasificación de Thomas B. Fitzpatrick" (Griffiths CEM, 2007). El primer estudio sobre la incidencia de psoriasis se realizó en el condado de Olmsted, Minnesota, en 1980. "La tasa de incidencia anual promedio bruta fue de 54.4 / 100.000 para hombres y 60.2 / 100.000 para mujeres". (Griffiths CEM, 2007) En los EE. UU "La psoriasis afecta aproximadamente al 2\% de la población, aunque se han informado tasas tan altas como el 4,6\%". (Lowes MA, 2007)

Existen tratamientos comunes que pueden llegar a aliviar los síntomas, pero no llegan a curarse.

\section{Presentación clínica de la enfermedad.}

Según Zhang P, la psoriasis es una enfermedad inflamatoria autoinmune de la piel, crónica común con un espectro de fenotipos clínicos y resulta de la interacción de factores genéticos, ambientales e inmunológicos. (2017)

En la actualidad se han determinado varios mecanismos patógenos subyacentes a la enfermedad, por lo que se ha descubierto diferentes terapias eficaces, que apuntan a mejorar la vida de los pacientes.

La psoriasis, es una de esas afecciones que abarca varios fenotipos clínicos distintos, que representan un aspecto dinámico, anatómico o cualitativo de la misma enfermedad. La presentación de la enfermedad no varía en cuanto el género de los pacientes, la mayoría de estos presenta síntomas de prurito, y varios casos llegan a destacar como punto de inicio, al cuero cabelludo.

Durante varios años "La enfermedad se clasifica según su aspecto clínico, determinando la morfología y su localización; tomando en cuenta así, a la clasificación propuesta por el Consejo Internacional de Psoriasis, que identifica cuatro formas principales de psoriasis: tipo de placa, guttata, GPP y eritrodermia, y varios subfotipos adicionales según su localización anatómica ( flexión, cuero cabelludo, palmas / plantas / uñas), tamaño (grande vs. pequeño) y grosor (grueso vs. delgado) de las placas, inicio (temprano vs. tardío) y actividad de la enfermedad (activo vs. estable)". (Oppmann B, Lesley R, 2000)

\section{Psoriasis tipo placa.}

La psoriasis de tipo placa, es "la forma más común de psoriasis y se caracteriza por su forma ovalada o irregular, rojas, de manera demarcadas, cubiertas por escamas plateadas. Las placas se localizan fundamentalmente en la superficie de los codos y rodillas, en el cuero cabelludo y en la parte inferior de la espalda". (Di Meglio P, 2014)

El término psoriasis en placa grande para Mrowietz $U$ especifica que " $(>3 \mathrm{~cm})$ describe placas rojas gruesas $(>0.75 \mathrm{~cm}$ ) bien delimitadas con escamas plateadas. La psoriasis en placa pequeña $(<3 \mathrm{~cm})$ se presenta con numerosas lesiones; las placas son más delgadas $(<0.75 \mathrm{~cm})$, de color 
rosado con una escala fina, y pueden estar bien definidas o fusionarse con la piel circundante". (2009)

Los cambios morfológicos de la enfermedad se acompañan con la evolución de una lesión hacia las placas, que pueden agrandarse con el tiempo, o simplemente permanecer estáticas.

\section{Psoriasis Guttata}

"La psoriasis guttata, del latín "gutta" para la caída de lágrimas, por lo que también se la denomina psoriasis en gota. Se caracteriza por múltiples placas pequeñas y escamosas que suelen aparecer alrededor del tronco y la parte superior de los brazos y los muslos". (Mrowietz U, 2009)

La psoriasis en gotas puede eliminarse completamente de manera espontánea o puede desaparecer tras el debido tratamiento. Sin embargo, algunas veces llega a volverse crónica o empeorar según el tipo de placa.

\section{Psoriasis pustulosa generalizada}

Este tipo de psoriasis es también conocido como psoriasis de von Zumbush, es una enfermedad mortal y muy rara, que se caracteriza por una inflamación sistémica y de la piel de manera generalizada.

Su presentación clínica se basa en la presencia de fiebre alta, fatiga y leucocitosis por neutrófilos. Este tipo de psoriasis se puede asociar al de tipo placa.

\section{Psoriasis Eritrodérmica}

"La psoriasis eritrodérmica, se denomina como el fenotipo más grave, que se caracteriza por un eritema difuso. En varias ocasiones los pacientes pueden llegar a presentar manifestaciones sistémicas, como hipotermia y edema de extremidades, así como a la mialgia, la fatiga y la fiebre". (Mrowietz U, 2009)

En la actualidad se ha determinado como posibles factores de riesgo para desarrollar la enfermedad, a las quemaduras solares, retiro de corticos esteroides sistémico e incluso el estrés emocional.

\section{Inmunología de la Psoriasis.}

La psoriasis es una enfermedad inflamatoria crónica de la piel mediada por células $\mathrm{T}$, que afecta a personas con predisposición genética y en la que ocurrirá una hiperplasia de la epidermis debida a la infiltración con células inmunes.

Para Nestle FO, Gaspari AA y Schröder JM, (2009) en "el cuadro inmunológico inflamatorio de la psoriasis intervienen, tanto elemento de la respuesta inmune innata, como de la respuesta adaptativa. Entre los primeros se encuentran las células dendríticas presentadoras de antígenos, los 
queratinocitos, los neutrófilos, macrófagos y las células NK (del inglés natural killer); entre los segundos, los linfocitos T CD4+ y CD8+”.

\section{Células NK}

Martin MP, menciona que "Las células NK pueden activar o inhibir la respuesta inmune a través de sus receptores KIRs (del inglés killer immunoglobulin-like). (23) El alelo HLA-Cw6 es el ligando natural para el receptor inhibitorio KIR2DL1 y es posible que la interacción entre ambos conduzca a una función aberrante de las células linfoides que contribuye a la inmunopatogenia de la psoriasis". (2002)

Por otro lado Mak RKH especifica que "Las células NK producen predominantemente IFN-a y pueden causar citólisis de diferentes células blanco. En las placas psoriásicas se observan niveles elevados de interleucinas, como las IL-2 e IL-15, que regulan la actividad NK”. (2009)

\section{Papel de las células presentadoras de antígenos y sus productos en la Psoriasis.}

Mrowietz U (2009) refiere que en las etapas iniciales y de exacerbación del psoriasis se observa una activación de las células dendríticas de la dermis y la epidermis. Estas células producen citosinas, como el factor de necrosis tumoral alfa (FNTa) y la IL-23, que promueven el desarrollo de las subpoblaciones de células T, Th1 y Th17, las cuales secretan mediadores que contribuyen a los cambios vasculares y epidérmicos presentes en la psoriasis.

Estudios con el empleo de inhibidores del FNTa en la psoriasis sugieren que este interviene en la regulación de la producción inicial de citosinas, en el desarrollo del proceso inflamatorio por las vías del interferón ganma (IFNa) y el transductor de señales y activador de transcripción (STAT, del inglés signal transducer and activator of transcription) y estimula la producción de quimosinas reguladoras de las células $\mathrm{T}$ y de las interacciones de las células dendríticas en la piel. (Lowes Ma, 2007).

Mak RKH, (2010) sostiene que En la fisiopatogenia del psoriasis intervienen 3 tipos de células dendríticas: las células de Langerhans, las células dendríticas dérmicas y las células dendríticas plasmacitoides. Al parecer, estas últimas constituyen un puente entre la respuesta inmune innata y la adaptativa en la patogenia del proceso inflamatorio del psoriasis.

Se ha evidenciado la participación de la célula de Langerhans, presentadora de antígeno, de ahí surge el concepto de enfermedad antígeno-dependiente. Los LT activados no reconocen lo propio, de allí surge el concepto de enfermedad autoinmune.

La psoriasis se sustenta en su base genética, su desequilibrio inmune, en la presentación antigénica, la intolerancia a lo propio, la liberación e interacción de citoquinas proinflamatorias, inmune moduladoras e inductoras de la proliferación vascular y de la proliferación epidérmica. 
Las células dendríticas plasmacitoides son importantes efectoras de la defensa antiviral en el sistema inmune innato a través de la secreción de grandes cantidades de IFN $\square$ en respuesta a estimulación viral por sus receptores TLR-7 y TLR-9. (Mak RKH, 2010)

El IFNa es un activador de la respuesta inmune innata que está presente en las lesiones de piel de los pacientes con psoriasis. Por otra parte, la estimulación continua con IFN $\square$ provoca en modelos animales una enfermedad inflamatoria en la piel similar al psoriasis y la administración de IFNa recombinante a pacientes con psoriasis para el tratamiento de infecciones virales o tumores puede empeorar la psoriasis. (Nestle FO, Conrad C y Funk J, Langeland, 1991)

\section{Psoriasis como enfermedad autoinmune}

Aunque las evidencias indican que la psoriasis es una enfermedad autoinmune dependiente de las células $\mathrm{T}$ y existen diferentes hipótesis para explicar la generación de autoinmunidad, aún no se ha definido bien el autoantígeno blanco. (Mak RKH, 2009)

Algunos estudios recientes sugieren que el péptido endógeno antimicrobiano LL37 que se expresa en los queratinocitos, de los que se libera en respuesta a daño o infección, puede combinarse con el ADN propio y activar las células dendríticas plasmacitoides con la subsiguiente producción de IFNa. Esto podría explicar cómo el ADN propio puede estimular autoinmunidad en la psoriasis. Lande R y Gregorio J (2007)

El aumento de la expresión del LL37 parece ser específico del psoriasis, ya que no se observa en la piel normal ni en otras enfermedades de la piel ni en la piel libre de lesiones de los pacientes con psoriasis. (Mak RKH, 2009)

Madonna S (2010) postula que existen algunas evidencias que señalan a los queratinocitos como el blanco directo de un grupo de citosinas que pueden regular algunas de sus propiedades biológicas y sus capacidades de diferenciación y migración. Guilloteau (2010) y otros identificaron que IL-17A, IL-22, oncostatin M, IL-1a, y el FNTa pueden actuar de forma sinérgica in vitro para generar un modelo inflamatorio de queratinocitos. En otro estudio en que se empleó IL-22 para generar Th17, se indujo un cuadro inflamatorio de piel en ratones, similar al del psoriasis.

Como expresa Fitch E (2007) las lesiones de piel y el líquido sinovial de los pacientes con artritis psoriásica son ricas en IL-6, IL-17 e IL-23, las cuales puede producir activación de factor B nuclear y estimular señales para la diferenciación celular.

Otra hipótesis que se ha invocado es la imitación molecular, de acuerdo con la cual los clones de células $\mathrm{T}$ generados originalmente en respuesta a la faringitis o tonsilitis estreptocócica son subsecuentemente reactivados en la piel por una epítrope de reacción cruzada de los queratinocitos epidérmicos hiperproliferativos, tales como la keratina 16 o la keratina 17. (Fry L, 1988)

Algunos investigadores sugieren que el genotipo de susceptibilidad al psoriasis puede conferir protección para la mortalidad en epidemias de infecciones por estreptococo, ya que los cambios 
en la respuesta inmune innata y adaptativa y la red de citosinas de las células Th 17 incrementan la eficiencia en la internalización del estreptococo. (Baker BS, 2006)

\section{Las células $\mathbf{T}$}

Entre los linfocitos $\mathrm{T}$, infiltrantes en las lesiones del psoriasis, se han identificado las subpoblaciones T helper 1 (Th1; CD4+), T citotóxicos (Tc1; CD8+), Th 17 y células T reguladoras. (Sugiyama H, 2005)

Johnston (2009) menciona que la psoriasis es una enfermedad tipo Th121 en la que existen niveles elevados de las citosinas de ese patrón, como el IFNa, el FNTa y la IL-12.

Por otra parte, las citosinas del patrón Th2 (IL-4, IL-5, IL-10) parecen ejercer un efecto protector contra la enfermedad. El uso terapéutico de IL-4 e IL-10 en pacientes con psoriasis produce una reversión del proceso inflamatorio. (Ghoreschi K, Thomas P, y Asadullah K, Docke W, 1999)

La psoriasis se considera actualmente un paradigma de enfermedad inflamatoria en la que desempeña un papel protagónico la subpoblación Th 17, lo que se conoce como el eje IL-23/ Th17. (Di Cesare A, 2009)

Las células T naive, bajo la acción del factor de crecimiento transformante beta (FCTB), la IL-6 y la IL-21, se transforman en células que expresan el factor de transcripción específica de linaje único, RORC variante 2 y los receptores de IL-23.

Después de la unión con la IL-23, estas células se transforman en células Th17. (Mak RKH, Hundhausen C y Di Cesare A, Di Meglio P, 2009)

Las células Th17 producen las citosinas proinflamatorias IL-17A, IL-17F, IL-22 e IL-26, las cuales activan los queratinocitos, estimulándolos a la hiperproliferación y producción de citosinas proinflamatorias, quimosinas y péptidos antimicrobianos, los que a su vez reclutan y activan otras células inmunes a la piel inflamada. (Langrish CL, 2005) De este modo se amplifica la respuesta inflamatoria que conduce a las manifestaciones clínicas de la enfermedad.

Una evidencia que sustenta el papel de las células Th17 en la psoriasis, es el aumento de las concentraciones de p40 (una subunidad común de la IL-12, IL-23 e IL-17) en la piel y articulaciones afectadas. (Piskin G, 2006)

De acuerdo con Oppmann B (2000) La IL-12 activa principalmente a las células Th1, mientras que la IL-23 interviene en el desarrollo de las células Th17.

En algunos estudios en humanos se ha detectado sobrexpresión de precursores de IL-23 (IL-23p19, IL-12p40) en el ARNm de piel; y producción de IL-23 en los sitios inflamados por células inmunes residentes en el tejido, como las células dendríticas y posiblemente los queratinocitos. Las células inmunes reclutadas al sitio de la inflamación también pueden producir IL-23. (Piskin G, 2006) 
Piskin G (2004) y Haider AS (2008) plantean otro hallazgo que refuerza el papel de la IL-23 en la patogenia del psoriasis es el tratamiento exitoso de esta entidad con productos que reducen los niveles de IL-23, como la ciclosporina A, la terapia ultravioleta y los agentes biológicos.

Como expresan Mak RKH (2009) y Sugiyama H (2005) en la psoriasis existen, además, defectos de la función y de la proliferación de las células T reguladoras. Estas células se caracterizan por su habilidad de suprimir la activación y proliferación de las células T efectoras CD4+ y CD8+, a través de mecanismos que requieren, tanto el contacto directo con las células presentadoras de antígeno, como de la liberación de IL-10 o del TGF- $\square$ 1. Los defectos de las células T supresoras en la psoriasis pueden conducir a un fallo en el control de la proliferación y activación de las células T patogénicas, lo que contribuye a la inflamación.

\section{Tratamiento}

No existe una norma fija de tratamiento para todos los pacientes, debiéndose decidir el tratamiento de una forma individualizada. Se dispone de tratamientos en los que se encuentran: la fototerapia, antiinflamatorios y anti proliferativos tópicos, sistemáticos y agentes biológicos.

El tratamiento tópico se utiliza más en pacientes con psoriasis leve-moderada llega a incluir corticoides, este es uno de los tratamientos más básicos, la elección de los corticoides dependerá de la localización de la lesión y su cronicidad; generalmente el trato con corticoides dependerá de la fototerapia y el trato sistemático. También se llegan a utilizar medicamentos con análogos de vitamina D que tienen la función anti proliferativa como el calcipotriol, que induce a la diferenciación de los queratinocitos; el tacalcitol, que tiene una función parecida al calcipotriol, pero es menos irritante y se utiliza en cara. Otro medicamento que se utiliza es el tazaroteno, una derivada de la vitamina A, que tiene una eficacia análoga a los de la vitamina D. (Valdes, D. M. 2012)

La terapia sistemática se usa para psoriasis moderada-grave y suele precisar un tratamiento agresivo. En estos fármacos se reduce la actividad inflamatoria de la enfermedad, mejorando los síntomas de la piel durante los brotes. Algunos de los medicamentos bloquean la acción de una proteína denominada fosfodiesterasa 4 (PDE4) implicada en la inflamación, al evitar que funcione correctamente la PDE4, se reduce la inflamación y otros síntomas de la psoriasis y artritis psoriásica.

La luz ultravioleta, tanto UVB como UVA se pueden usar en el tratamiento del psoriasis, tiene la propiedad de ralentizar la reproducción del queratinocito y de relajar la actividad de las células inflamatorias.

En los agentes biológicos estos medicamentos llegan a presentar un perfil de seguridad más eficaces y favorables que los sistemáticos; los efectos adversos son más leves. Muestran mejores resultados que tratamientos con medicamentos sistemáticos, son mucho más tolerables y menor toxicidad. Los más utilizados son infliximab, un anticuerpo monoclonal quimérico que se une a 
TNF $\alpha$ y se usa en pacientes que necesitan unas respuestas rápidas para combatir la rapidez de la psoriasis; etanercept, proteína de fusión humana formada por el receptor TNF $\alpha$ fusionado con la fracción Fc de la IgG humana que llega a inactivar el TNF $\alpha$; ustekinumab, anticuerpo monoclonal humano dirigido contra la subunidad p40 de IL-12 e IL-23, es una inyección que detiene algunos de los síntomas de la psoriasis; y adalimumab, es un anticuerpo monoclonal recombinante IgG1 humano que se une con elevada afinidad al TNFa, reduce los síntomas, inhibe la progresión del daño estructural y mejora su función física en los pacientes adultos.

\section{Conclusiones.}

Con respecto a la Inmunología de la Psoriasis, se puede resumir así:

- El componente básico de la lesión psoríasica es la epidermopoyesis anormalmente aumentada.

- Está acompañada de anomalías (bioquímicas e inmunológicas) del queratinocito.

- Las anomalías queratinocitarias se deben a los factores inmunitarios, particularmente a las citoquinas producidas por el linfocito TCD4 disfuncional.

- Los problemas inmunológicos están bajo la dependencia de genes de predisposición y de antígenos y/o superantígenos que estimulan los linfocitos.

- Por último, las definiciones patogénicas de psoriasis son múltiples pero cada vez son más numerosas las que sostienen el rol preponderante del linfocito $\mathrm{T}$, sin poder descartar el rol de otros factores sobre la enfermedad

\section{Referencias bibliográficas.}

Asadullah K, Docke WD, Ebeling M, Friedrich M, Belbe G, Audring H, et al. (1999). Interleukin 10 treatment of psoriasis: clinical results of a phase 2 trial. Arch Dermatol, 135(2):187-92.

Baker BS, Laman JD, Powles A, van der Fits L, Voerman JS, Melief MJ, et al. (2006). Peptidoglycan and peptidoglycan-specific Th1 cells in psoriatic skin lesions. J Pathol, 209(2):174-81.

Bharti Vidyappeth, M. P. (2011). Psoriasis: A comprehensive review. International Journal of pharmacy \& life sciences, 857-877.

Cimmino, M. (2007). Epidemiology of psoriasis and psoriatic arthritis, 59(1):19-24.

Dermatology, T. s. (2016). What is psoriasis. the society for pediatric dermatology.

Di Cesare A, Di Meglio P, Nestle FO.(2009). The IL-23/Th17 Axis in the Immunopathogenesis of Psoriasis. J Invest Dermatol, 129(6):1339-50. 
Di Meglio P, Villanova F, Nestlé FO. (2014). Psoriasis. Cold Spring Harb Perspect Med, 4(8): 015-354.

Fitch E, Harper E, Skorcheva I, Kurtz SE, Blauvelt A.(2007). Pathophysiology of psoriasis: Recent advances on IL-23 and TH17 cytokines. Curr Rheumatol Rep, 9(6):461-7.

Fry L.(1988).Psoriasis. Br J Dermatol, 119(4):445-61.

Funk J, Langeland T, Schrumpf E, Hanssen LE.(1991). Psoriasis induced by interferonalpha. Br J Dermatol, 125(5):463-5.

Gaspari AA.(2006). Innate and adaptive immunity and the pathophysiology of psoriasis. J Am Acad Dermatol, 54(3 Suppl 2):S67-80.

Ghoreschi K, Thomas P, Breit S, Dugas M, Mailhammer R, van Eden W, et al.(2003). Interleukin4 therapy of psoriasis induces $\mathrm{Th} 2$ responses and improves human autoimmune disease. Nat Med, 9(1):40-6.

Griffiths CEM, C. E. (2007). A classification of psoriasis vulgaris according to phenotype. $\mathrm{Br} \mathbf{J}$ Dermatol.

Guilloteau K, Paris I, Pedretti N, Boniface K, Juchaux F, Huguier V, et al. Skin inflammation induced by the synergistic action of IL-17A, IL-22, oncostatin M, IL-1 $\square$, and TNF- $\square$ recapitulates some features of psoriasis. J Immunol. 2010 Mar 24;184:5263-70.

Haider AS, Lowes MA, Suárez-Farinas M, Zaba LC, Cardinale I, Khatcherian A, et al. Identification of cellular pathways of "type 1," Th17 T cells, and TNF- and inducible nitric oxide synthaseproducing dendritic cells in autoimmune inflammation through pharmacogenomic study of cyclosporine A in psoriasis. J Immunol. 2008 Feb 1;180(3):1913-20.

Johnston A, Gudjonsson JE, Sigmundsdottir H, Love TJ, Valdimarsson H. Peripheral blood T cell responses to keratin peptides that share sequences with streptococcal M proteins are largely restricted to skin-homing CD8(+) T cells. Clin Exp Immunol. 2004 Oct;138(1):83-93. natural selection of psoriasis revisited. Br J Dermatol. 2009 May;160(5):929-37.

Juliana L Basko-Plluska, V. P.-R. (2012). Psoriasis: epidemiology, natural history, and differential diagnosis. Dove Press journal.

Lande R, Gregorio J, Facchinetti V, Chatterjee B, Wang YH, Homey B, et al. Plasmacytoid dendritic cells sense self-DNA coupled with antimicrobial peptide. Nature. 2007 Oct 4;449(7162):564-9. 
Langrish CL, Chen Y, Blumenschein WM, Mattson J, Basham B, Sedgwick JD, et al. IL 23 drives a pathogenic $\mathrm{T}$ cell population that induces autoimmune inflammation. J Exp Med. 2005 Jan 17;201(2):233-40.

Lowes MA, Bowcock AM, Krueger JG. Pathogenesis and therapy of psoriasis. Nature. 2007 Feb 22;445(7130):866-73.

Ma HL, Liang S, Li J, Napierata L, Brown T, Benoit S, et al. IL-22 is required for Th17 cellmediated pathology in a mouse model of psoriasis-like skin inflammation. J Clin Invest. 2008 Feb;118(2):597-607.

Madonna S, Scarponi C, Sestito R, Pallotta S, Cavani A, Albanesi C. The IFNgamma- dependent suppressor of cytokine signaling 1 promoter activity is positively regulated by IFN regulatory factor-1 and Sp1 but repressed by growth factor independence-1b and Kruppel like factor-4 and is dysregulated in psoriatic keratinocytes. J Immunol. 2010 Aug 15;185(4):2467-81.

Mak RKH, Hundhausen C, Nestle FO. Progress in understanding the immunopathogenesis of psoriasis Actas Dermosifiliogr. Author manuscript; available in PMC 2010 October 20. Published in final edited form as: Actas Dermosifiliogr. 2009 Dec; 100(Suppl 2):2-13. PMC2957885.

Martin MP, Nelson G, Lee JH, Pellett F, Gao X, Wade J, et al. Cutting edge: susceptibility to psoriatic arthritis: influence of activating killer Ig-like receptor genes in the absence of specific HLA-C alleles. J Immunol. 2002 Sep 15;169(6):2818-22.

Mrowietz U, Reich K. Psoriasis-new insights into pathogenesis and treatment. Dtsch Arztebl Int. 2009 Jan;106(1-2):11-8, quiz 19. PMID19564982. PMCID: PMC2695319.

Nestle FO, Conrad C, Tun-Kyi A, Homey B, Gombert M, Boyman O, et al. Plasmacytoid predendritic cells initiate psoriasis through interferon-alpha production. Exp Med. $2005 \mathrm{Jul}$ 4;202(1):135-43.

Nestle FO, Kaplan DH, Barker J. Mechanisms of disease: Psoriasis. N Engl J Med. 2009 Jul 30;361(5):496-509. PubMed: 19641206.

Oppmann B, Lesley R, Blom B, Timans JC, Xu Y, Hunte B, et al. Novel p19 protein engages IL$12 \mathrm{p} 40$ to form a cytokine, IL-23, with biological activities similar as well as distinct from IL-12. Immunity. 2000 Nov;13(5):715-25.

Piskin G, Sylva-Steenland RM, Bos JD, Teunissen MB. In vitro and in situ expression of IL-23 by keratinocytes in healthy skin and psoriasis lesions: enhanced expression in psoriatic skin. J Immunol. 2006 Feb 1;176(3):1908-15. 
Piskin G, Tursen U, Sylva-Steenland RM, Bos JD, Teunissen MB. Clinical improvement in chronic plaque-type psoriasis lesions after narrow-band UVB therapy is accompanied by a decrease in the expression of IFN-gamma inducers- IL-12, IL-18 and IL-23. Exp Dermatol. 2004 Dec;13(12):764-72.

Schröder JM, Reich K, Kabashima K, Liu FT, Romani N, Metz M, et al. Who is really in control of skin immunity under physiological circumstances-lymphocytes, dendritic cells or keratinocytes? Exp Dermatol. 2006 Nov;15(11):913-29.

Sugiyama H, Gyulai R, Toichi E, Garaczi E, Shimada S, Stevens SR, et al. Dysfunctional blood and target tissue CD4+ CD25 high regulatory T cells in psoriasis: mechanism underlying unrestrained pathogenic effector T cell proliferation. J Immunol. 2005 Jan 1;174(1):16473.

Valdes, D. M. (2012). Inmunopatogenia de la psoriasis. Impacto en las manifestaciones y el tratamiento de la enfermedad. Revista Cubana Hematol-Inmunol.

Van de Kerkhof PCM, Nestle FO. Psoriasis. En Dermatología. Elsevier, Amsterdam. 2012.

Zhang P, Wu MX. Una revisión clínica de la fototerapia para la psoriasis.Láseres Med Sci. 2017; 33 (1): 173-180. 


\section{PARA CITAR EL ARTÍCULO INDEXADO.}

Jorge Cañarte Alcivar., Vielka Jiménez García., Johan Muñoz Puertas., Ambar Luzardo Robles. \& Josue Delgado Villafuerte. (2019). Inmunopatogenia del psoriasis, evolución de la enfermedad, presentación clínica y tratamiento. Revista electrónica Ciencia Digital 3(2), 78-97. Recuperado desde:

http://cienciadigital.org/revistacienciadigital2/index.php/CienciaDigital/article/view/263/567

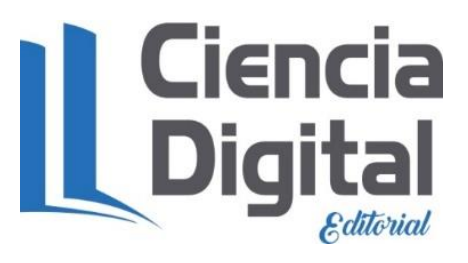

El artículo que se publica es de exclusiva responsabilidad de los autores y no necesariamente reflejan el pensamiento de la Revista Ciencia Digital.

El artículo queda en propiedad de la revista y, por tanto, su publicación parcial y/o total en otro medio tiene que ser autorizado por el director de la Revista Ciencia Digital.
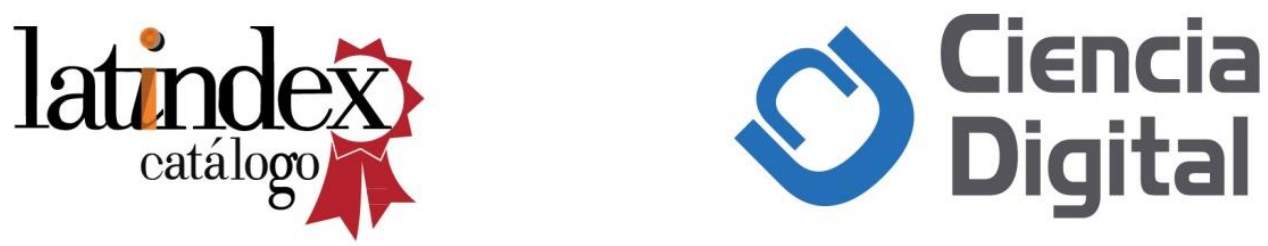